\title{
Polarization-maintaining optical microfiber
}

\author{
Yongmin Jung*, Gilberto Brambilla, and David J. Richardson \\ Optoelectronics Research Centre, University of Southampton, Southampton, SO17 1BJ, UK \\ *Corresponding author: ymj@orc.soton.ac.uk
}

\begin{abstract}
We have successfully demonstrated a polarization-maintaining (PM) fused silica microfiber by adiabatically tapering a conventional polarization-maintaining fiber. Compared to standard single mode microfibers, the proposed PM microfibers exhibit robust polarization preserving characteristics under the presence of external perturbations such as bending. A polarization extinction ratio of $16 \mathrm{~dB}$ is typically obtained through the device with a corresponding excess loss of 0.2dB. (C2010 Optical Society of America
\end{abstract}

OCIS Codes: (060.2420) Fiber, polarization-maintaining; (260.1440) Birefringence; (220.4000) Microstructure fabrication; (220.4241) Nanostructure fabrication

With recent advances in micro- and nanophotonics, optical micro-/nanofibers with diameters close to the wavelength of guided light have attracted considerable interest as promising building blocks for a variety of photonic applications [1-4]. One of the most important issues is the fabrication of polarization maintaining (PM) microfibers since unwanted changes in the polarization of the propagating signal can result in a considerable deterioration in system reliability and performance for many applications. There are two principal approaches to produce 
PM microfibers. One is to use non-circular fibers such as side-flat fibers [5, 6]. By breaking the circular symmetry observed in conventional optical fiber tapers, a strong form birefringence can be achieved in the taper waist region. The second approach involves the use of commercial polarization maintaining fibers (PMFs) such as panda and bow-tie fibers to preserve the input polarization state. By carefully controlling the parameters of microfiber manufacture, it should be possible to minimize the diffusion of dopants in the tapered region and maintain both the internal stress and the refractive index profiles as required to preserve polarization during propagation through the device.

In this paper, a commercial panda fiber (Nufern Inc. PM 1550HP) was employed as a host fiber to preserve the plane of polarization of the light and was tapered into a very thin waist $(\sim 1 \mu \mathrm{m})$ microfiber. The optical properties of the PM microfiber were examined by observing the polarization extinction ratio and the state of polarization at the microfiber output. The PM microfiber should find use in many micro-/nanophotonics applications and devices such as highquality micro-resonators, tight focusing optical microprobes, high performance optical sensors and microlasers amongst others.

Figure 1(a) shows the schematic configuration of the proposed PM microfiber. A short section of panda fiber was heated and stretched into a very thin microfiber similar to previous wellestablished bi-conical fiber tapers [7]. In the adiabatic taper transition, the local fundamental core mode $\left(L P_{01}{ }^{\text {core }}\right)$ is continuously mode converted to a guided cladding mode $\left(L P_{01}{ }^{\text {clad }}\right)$ in the taper waist by the down-taper and is then coupled back into the fundamental core mode $\left(L P_{01}{ }^{\text {core }}\right)$ of the fiber by the up-taper [8]. In this type of polarization preserving fiber stress rods induce tensile stress across the core and cause a difference in the refractive indices between the linearly polarized components that are parallel to the fast and slow axes as defined by the internal stress 
field. Polarised light launched on each axis of a PM fiber remains on that axis as it propagates through the fiber due to the resulting difference in propagation constants: even when the fiber is bent or twisted. The main focus of this study is to establish how well the input polarization state is preserved during the tapering process. First, the taper cross section was inspected under an optical microscope using a conventional cleaving tool (diamond scribe). As shown in Fig. 1(b), the fiber geometry is well preserved and the stress applying parts are clearly discernible even when the outer diameter is less than $10 \mu \mathrm{m}$.

To investigate the modal guidance, in-situ transmission spectra of the PM microfiber were recorded for various outer diameters during the tapering process. An incoherent white light source and an optical spectrum analyzer were used to measure the spectral characteristics of the PM microfiber. Figure 2 shows the spectral output of tapered PM fibers for different outer diameters (O.D.) in the uniform waist region. Just as in similar previous experiments on highorder mode filtering in a standard telecom fiber [8], the transition region is adiabatic for the fundamental mode $\left(\lambda>\lambda_{\text {cutoff }}=1295 \mathrm{~nm}\right)$ but non-adiabatic for the higher-order core mode $(\lambda<1295 \mathrm{~nm})$. Note that for the $1 \mu \mathrm{m}$ PM microfiber there is no higher-order mode cut-off and the optical loss for the fundamental mode due to the taper is less than $0.2 \mathrm{~dB}$ at $\lambda=1.55 \mu \mathrm{m}$.

To verify the polarization maintaining property of PM microfibers, we compared their properties with standard single mode (SM) microfibers in terms of polarization extinction ratio (PER). A cross-polarizer method $[9,10]$ was implemented to measure the PER according to which polarized light is launched into the core of the constituent fiber through a polarizer whose polarization axis is aligned to one of the principal axes of the PM fiber. The output light is then 
passed through an analyzer and the proportion of the output power that is linearly polarized along both the launch axis $\left(P_{\max }\right)$ and the orthogonal axis $\left(P_{\min }\right)$ is determined. The PER is defined as $\mathrm{PER}=10 \log _{10}\left(P_{\max } / P_{\min }\right)$. To achieve a good measurement accuracy, we chose a polarized broadband amplified spontaneous emission (ASE) source with a bandwidth of up to $60 \mathrm{~nm}$ and whose output polarization is accurately aligned to the principal polarization axes of the input PM fiber.

Prior to the main experiment, we tested the polarization properties of the host fibers (both standard SMF and PMF). As shown in Fig. 3(a), a short length (4m) of telecom SMF can exhibit a high PER $(\sim 30 \mathrm{~dB})$ at a certain polarization launch angle, where one of the birefringent axes is well aligned with the polarization direction of the incident light [11, 12]. However, transmission in the SMF was easily changed by external perturbations such as bending and twisting and the PER value deteriorated significantly. For the PMF, when stress was applied, the PER with respect to input polarization angle showed good polarization maintaining characteristics with significant tolerance to bending induced stress, as shown in Fig. 3(b). In order to explore the polarization properties of microfibers, both host fibers (SMF, PMF) were adiabatically tapered down to a waist diameter of $1 \mu \mathrm{m}$. As depicted in Fig. 3(c) and 3(d), the PER for the SM microfiber was $24 \mathrm{~dB}$, whereas for the PM microfiber it fell to $16 \mathrm{~dB}$. The decrease in the PER is possibly due to scattering phenomena in the taper waist that depolarize the propagating light. In all cases the PER is large enough to maintain the polarized state in the fiber and the PER in the PM microfiber can be increased with index-matched stress applying parts [13, 14]. Note that when manually applying bend perturbations to both microfibers the PM microfiber shows stable polarization maintaining characteristics under all bend and pressure conditions tested. However, the PER in the SM microfiber is easily changed by these factors and drops to somewhere around 
3dB. Therefore both SM and PM microfibers exhibits a good polarization maintaining capability in stable environmental situations. However, in the presence of bends and stresses, the PM microfiber exhibits robust PM characteristics. Therefore the PM microfiber allows for linearly polarized light launched along the proper axis to travel long distances without any change in polarization state.

Additional experiments were performed with a polarization analyzer [11]. The state of polarization (SOP) trace was measured under different bending conditions for both standard fibers (SMF, PMF) and microfibers (SM microfiber and PM microfiber). As can be seen in Fig. 4(a), the entire Poincare sphere was covered by the trace, which means that the SOP of the SMF is easily affected by external disturbances. By comparison, the SOP trace at the output of the PMF was localized to a certain point and did not change. The small circle on the Poincare sphere is due to a small misalignment of the direction of the linear polarized beam relative to the input PM fiber axis and can be minimized by more precise alignment. These different polarization characteristics for external perturbations are also observed in their microfibers with diameters close to the wavelength of guided light. The output from the PM microfiber is stable linearly polarized light with a PER $>16 \mathrm{~dB}$, whereas the SOP of the SM microfiber is unpredictable in the presence of external perturbations. This is simply a manifestation of the fact that the fabricated PM microfiber was stable against external perturbations and the relatively small excursions show that the polarization state was fairly stable over that entire measurement time.

In summary, we have studied the polarization maintaining properties of the proposed PM microfiber in terms of both PER and stability of output SOP and compared these with those of 
standard SM microfibers. Both forms of microfiber exhibit a good capability to maintain linear polarization. However, under the presence of external perturbations such as bending, the PM microfibers consistently maintain the light polarization state, while the polarization maintaining capabilities of SM microfibers are deteriorated. Therefore, PM microfibers have great potential to introduce an additional degree of polarization control/stability in micro-/nanophotonics applications.

The authors thank the Engineering and Physical Sciences Research Council UK (EPSRC) for financial support; GB gratefully acknowledges the Royal Society (London, UK) for his University Research Fellowship. 


\section{References}

1. L. Tong, R. R. Gattass, J. B. Ashcom, S. He, J. Lou, M. Shen, I. Maxwell and E. Mazur, “Subwavelength-diameter silica wires for low-loss optical wave guiding,” Nature 426, 816 (2003).

2. G. Brambilla, F. Xu, P. Horak, Y. Jung, F. Koizumi, N. P. Sessions, E. Koukharenko, X. Feng, G. S. Murugan, J. S. Wilkinson, and D. J. Richardson, “Optical fiber nanowires and microwires: fabrication and applications,” Adv. Opt. Photon. 1, 107 (2009).

3. Limin Tong, "Brief introduction to optical microfibers and nanofibers," Front. Optoelectron. China 3, 54 (2010).

4. G. Brambilla, “Optical fibre nanowires and microwires: a review,” J. Opt. 12, 043001 (2010).

5. Yongmin Jung, Gilberto Brambilla, Kyunghwan Oh, and David J. Richardson, "Highly birefringent silica microfiber,” Opt. Lett. 35, 378 (2010).

6. Haifeng Xuan, Jian Ju, and Wei Jin, "Highly birefringent optical microfibers,” Opt. express 18, 3828 (2010).

7. G. Brambilla, F. Koizumi, X. Feng, and D. J. Richardson, "Compound-glass optical nanowires,” Electron. Lett. 41, 400 (2005).

8. Yongmin Jung, Gilberto Brambilla and David J. Richardson, "Broadband single-mode operation of standard optical fibers by using a sub-wavelength optical wire filter," Opt. Express. 16, 14661 (2008).

9. Denis Penninckx and Nicolas Beck, "Definition, meaning, and measurement of the polarization extinction ratio of fiber-based devices,” Appl. Opt. 44, 7773 (2005). 
10. Tim T. Aalto, Mikko Harjanne, Markku Kapulainen, "Method for the rotational alignment of polarization-maintaining optical fibers and waveguides,” Opt. Eng. 42, 2861 (2003).

11. Gregory D. VanWiggeren and Rajarshi Roy, “Transmission of linearly polarized light through a single-mode fiber with random fluctuations of birefringence,” Appl. Opt. 38, 3888 (1999).

12. S. C. Rashleigh, W. K. Burns, R. P. Moeller, and R. Ulrich, "Polarization holding in birefringent single-mode fibers,” Opt. Lett. 7, 40 (1981).

13. Itaru Yokohama, Kazunori Chida, and Juichi Noda, "Low excess loss conditions of polarization-maintaining fiber couplers,” Appl. Opt. 27, 4807 (1988).

14. Y. Anjan and S. Habbel, "Environmental performance of fused PM fiber couplers for fiber gyro application,” IEEE Photon. Technol. Lett. 3, 578 (1991). 


\section{References}

1. L. Tong, R. R. Gattass, J. B. Ashcom, S. He, J. Lou, M. Shen, I. Maxwell and E. Mazur, Nature 426, 816 (2003).

2. G. Brambilla, F. Xu, P. Horak, Y. Jung, F. Koizumi, N. P. Sessions, E. Koukharenko, X. Feng, G. S. Murugan, J. S. Wilkinson, and D. J. Richardson, Adv. Opt. Photon. 1, 107 (2009).

3. L. Tong, Front. Optoelectron. China 3, 54 (2010).

4. G. Brambilla, J. Opt. 12, 043001 (2010).

5. Y. Jung, G. Brambilla, K. Oh, and D. J. Richardson, Opt. Lett. 35, 378 (2010).

6. H. Xuan, J. Ju, and W. Jin, Opt. express 18, 3828 (2010).

7. G. Brambilla, F. Koizumi, X. Feng, and D. J. Richardson, Electron. Lett. 41, 400 (2005).

8. Y. Jung, G. Brambilla and D. J. Richardson, Opt. Express. 16, 14661 (2008).

9. D. Penninckx and N. Beck, Appl. Opt. 44, 7773 (2005).

10. T. T. Aalto, M. Harjanne, M. Kapulainen, Opt. Eng. 42, 2861 (2003).

11. G. D. VanWiggeren and R. Roy, Appl. Opt. 38, 3888 (1999).

12. S. C. Rashleigh, W. K. Burns, R. P. Moeller, and R. Ulrich, Opt. Lett. 7, 40 (1981).

13. I. Yokohama, K. Chida, and J. Noda, Appl. Opt. 27, 4807 (1988).

14. Y. Anjan and S. Habbel, IEEE Photon. Technol. Lett. 3, 578 (1991). 


\section{List of Figure Captions}

Fig. 1. (a) Schematic of an adiabatically tapered PM microfiber, (b) microscope images of the fiber cross section along the taper.

Fig. 2. Transmission spectra of the proposed PM microfiber

Fig. 3. Polarization extinction ratios with respect to input polarizer angle for (a) SMF, (b) PMF, (c) SM microfiber and (d) PM microfiber.

Fig. 4. Polarization mapping onto the Poincare' sphere with respect to some bending conditions for (a) SMF, (b)PMF, (c) SM microfiber and (d) PM microfiber 


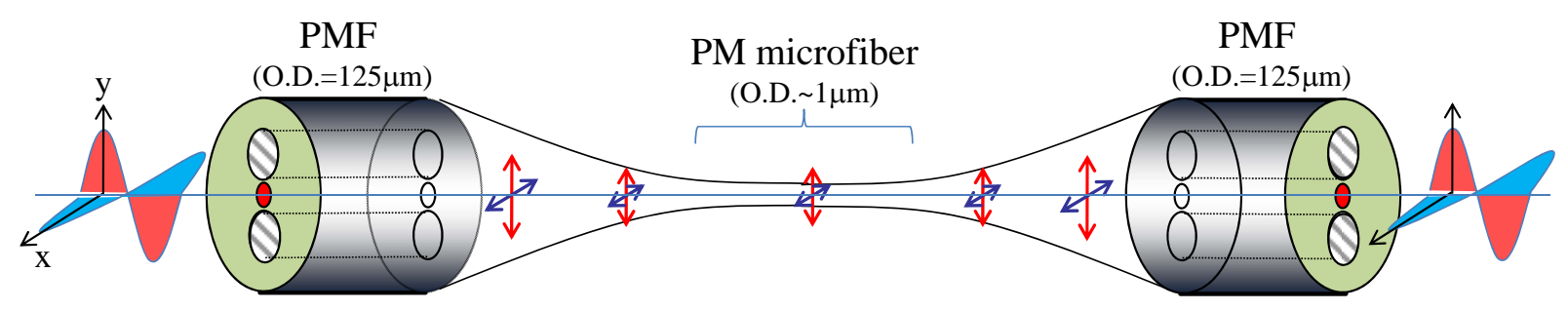

(a)

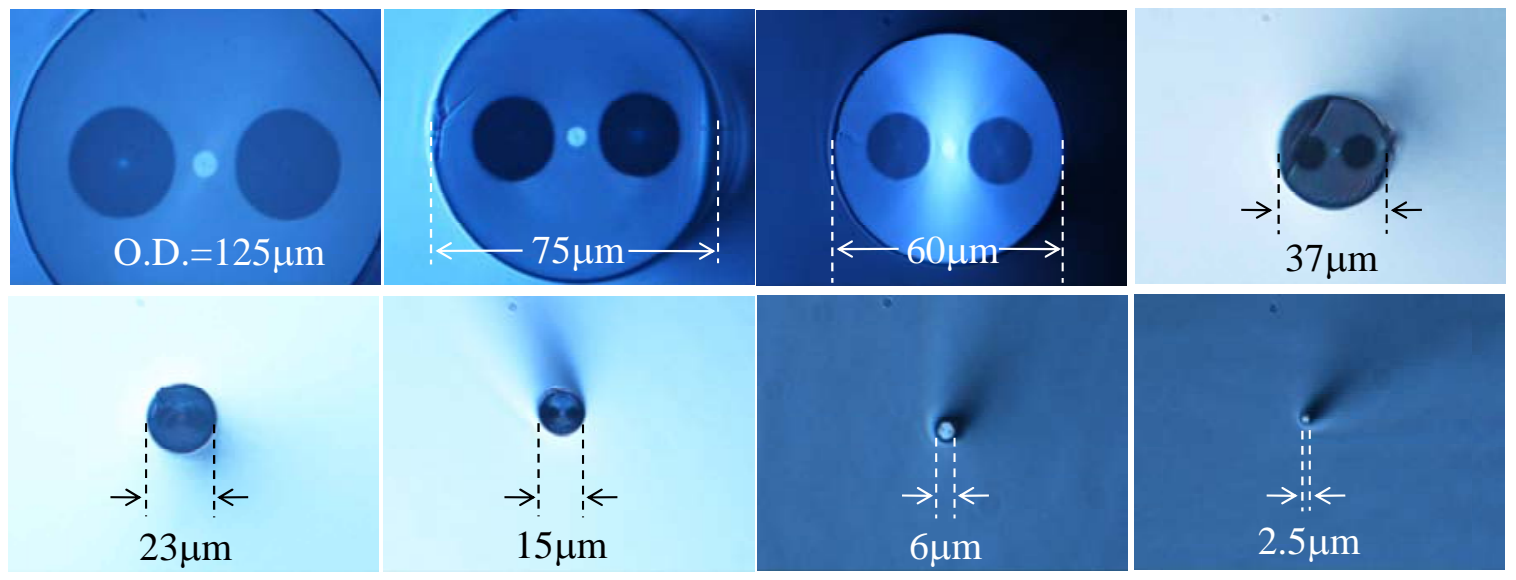

(b)

Fig. 1. Y. Jung, et al, Optics Letter. 


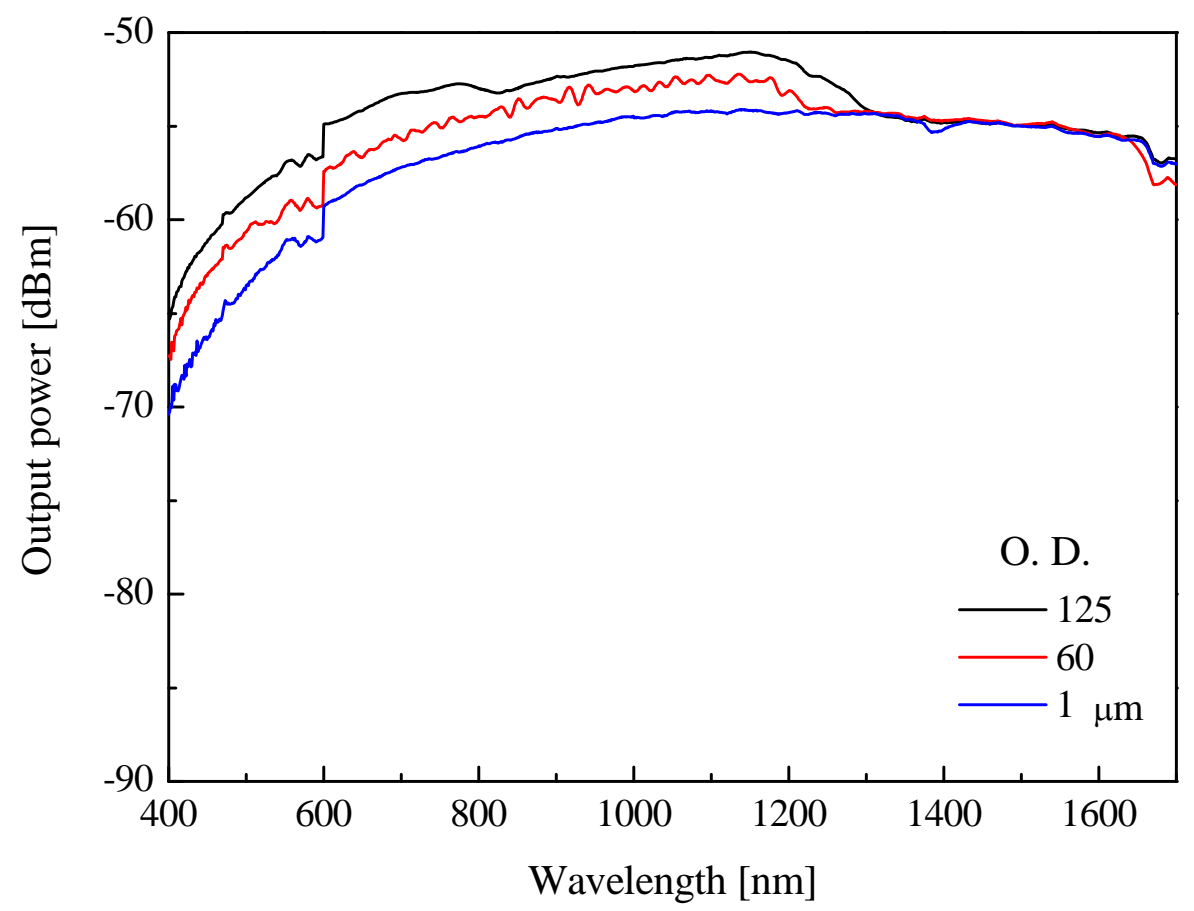

Fig. 2. Y. Jung, et al, Optics Letter. 


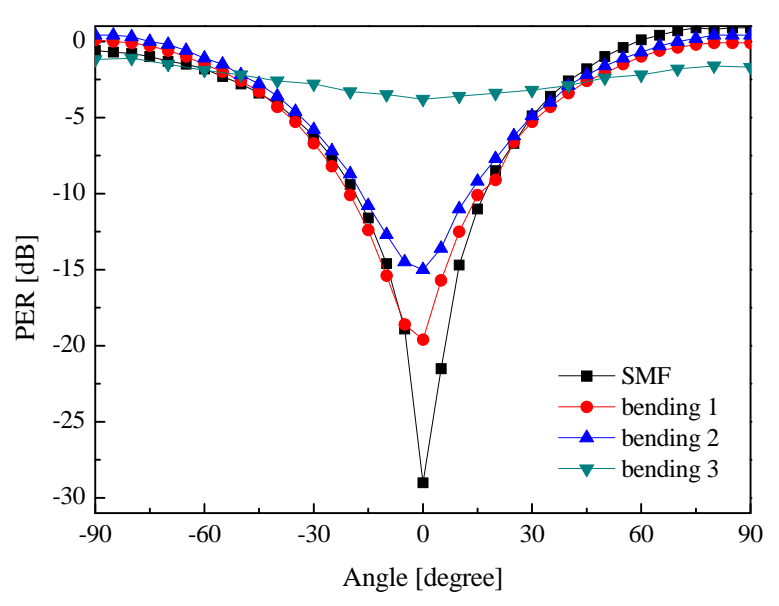

(a)

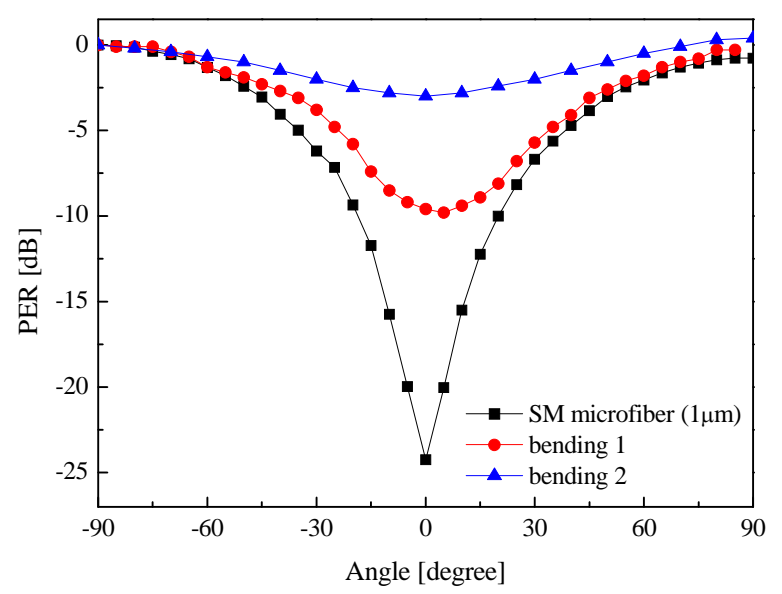

(c)

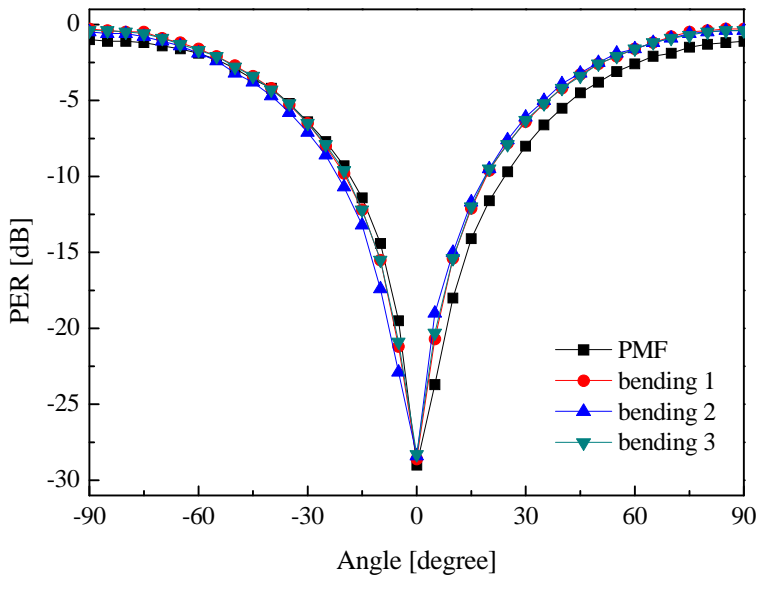

(b)

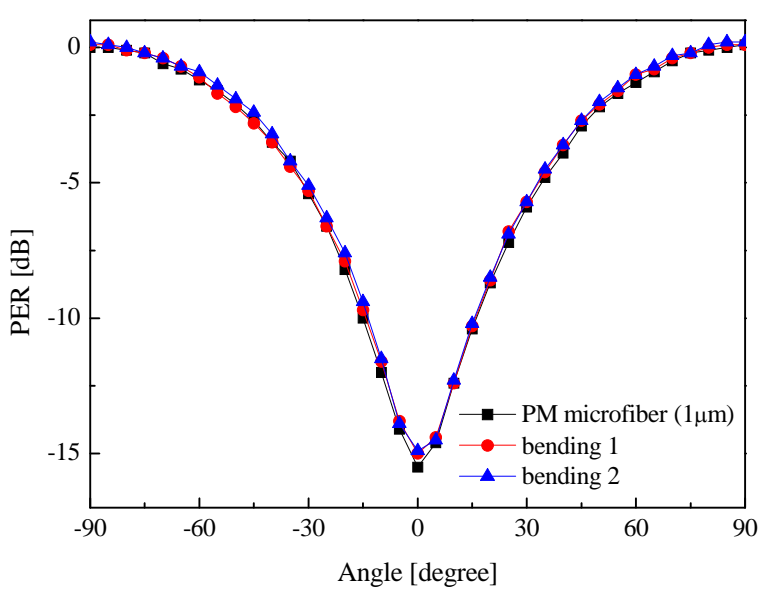

(d)

Fig. 3. Y. Jung, et al, Optics Letter. 


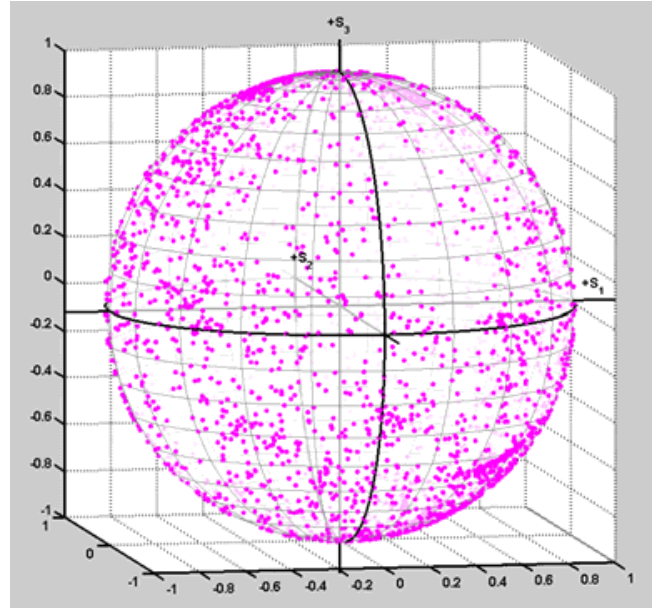

(a)

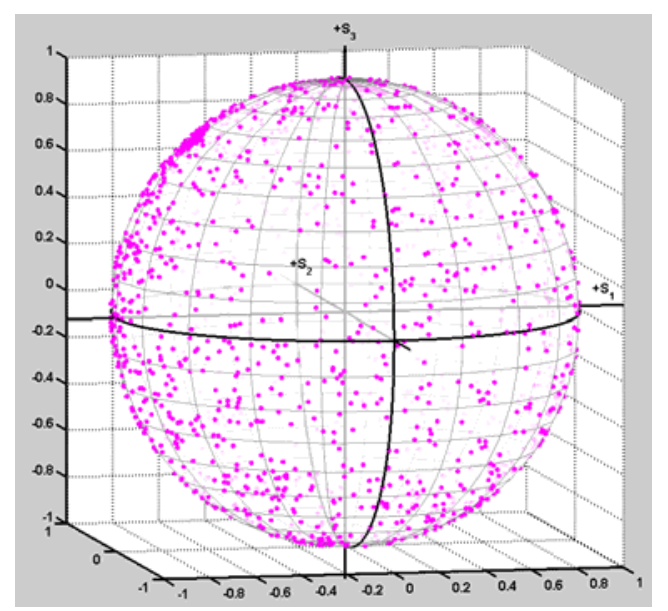

(c)

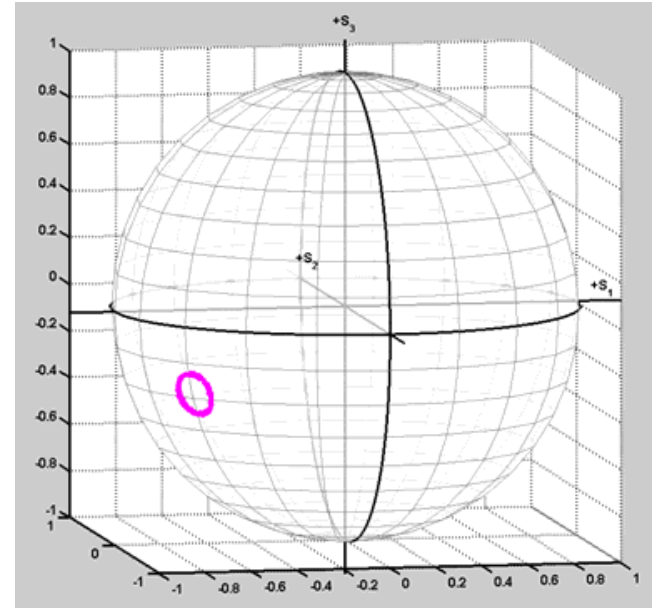

(b)

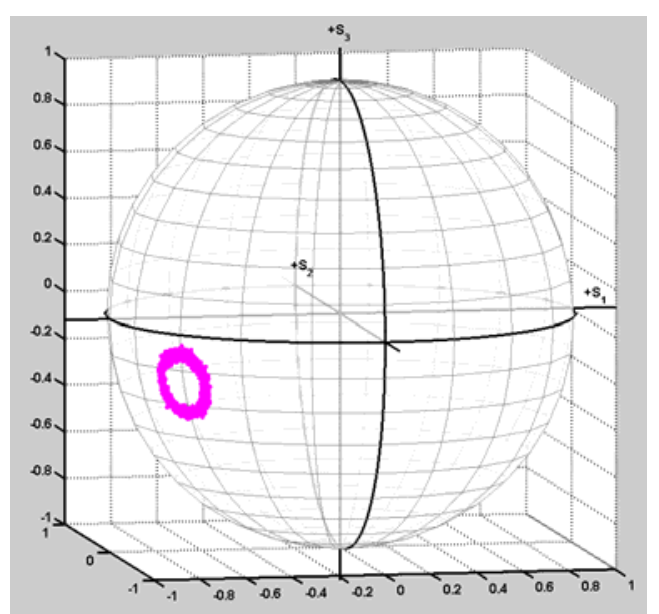

(d)

Fig. 4. Y. Jung, et al, Optics Letter. 